\title{
MALIGNANT MELANOMA IN THE ANTERIOR REGION OF MAXILLARY GINGIVA: A RARE CASE REPORT
}

Rajesh Twyana ${ }^{1}$, Zhang Hong yi $^{2}$, Jiang Wen ${ }^{3}$ Saroj Kumar Suwal ${ }^{4}$

\begin{abstract}
Malignant melanoma is extremely rare neoplasm arising from uncontrolled growth of melanocytes, accounting for $0.5 \%$ of all oral malignancies. It has a great tendency to metastasize and locally invade tissues more readily than any other malignant tumor of the oral cavity. It present as the rare oral malignant condition, asymptomatic and poor prognosis. The necessity of a highly specialized treatment is factors that should be seriously considered by the involved health care provider. Herein, we report a rare and interesting case of oral malignant melanoma of the maxillary anterior gingiva, which was clinically and histo-pathologically diagnosed with a brief review of literature, has been discussed.
\end{abstract}

KEYWORDS: Gingiva, melanocytes, Metastasis, Oral melanoma, Recurrence

1. Post Graduate Resident, Department of Oral \& Maxillofacial Surgery, The People's First Hospital of Jingzhou, Yangtze University, Jingzhou, Hubei,PR of China

2. Professor, Department of Oral \& Maxillofacial Surgery, The People's First Hospital of Jingzhou, Yangtze University, Jingzhou, Hubei,PR of China

3. MDS, Department of Oral \& Maxillofacial Surgery, The People's First Hospital of Jingzhou ,Yangtze University, Jingzhou, Hubei,PR of China

4. Post Graduate Resident, Department of Orthopedics, The People's First Hospital of Jingzhou, Yangtze University, Jingzhou, Hubei,PR of China

\author{
For Correspondence \\ Prof. Zhang Hong yi,Ph.D \\ Department of Oral and Maxilliofacial Surgery, \\ The People's First Hospital of Jingzhou, Yangtze \\ University, Jingzhou, Hubei ,PR of China, \\ E-mail:zhy88008@sina.com
}




\section{INTRODUCTION}

Melanoma is a malignant neoplasm of melanocytic origin that arises from a benign melanocytic lesion .Oral malignant melanoma (OMM) was first described by Weber in 1859.[1] Malignant melanoma of the oral cavity is an extremely rare tumor arising from the uncontrolled growth of melanocytes found in the basal layer of the oral mucous membranes. ${ }^{1,2}$ Nearly $80 \%$ of oral melanomas arise in the mucosa of the upper jaw, with the majority occurring on keratinizing mucosa of the palate and alveolar gingivae.[2] It occurs slightly more often in males 2.8:1 male to female ratio) and the age range is from. 20-83 years' with an average age of 56 years.

This case reports of OMM of the maxillary anterior gingiva without cervical lymph node metastasis, which has been discussed with detailed investigations such as biochemical; histopathology, ultrasound and contrast enhanced computed tomography (CECT) to emphasize the necessity for early diagnosis and treatment of this lesion.

\section{Case Report}

A 59-year-old male reported to the Department of Oral and Maxillofacial Surgery, The people's first hospital of Jingzhou, Yangtze University, Jingzhou, Hubei ,PR of China, with a complaint of bluish black discoloration in the upper right gums in relation to the front teeth since 3 months, which started as a pin point lesion, progressed gradually. He gave no history of any systemic illness or trauma to the head and neck region .General physical examination was insignificant and his vital signs were under limits. Extraoral examination revealed no any lymph node was palpable .On intraoral examination, a diffuse, sessile and asymptomatic swelling, with a smooth surface and of bluish black color was observed on the maxillary attached gingiva with in relation to right upper central incisor to canine about $3 \times 3 \mathrm{~cm}$ in size which was elevated and well-defined. [Figures1]. Based on clinical appearance, pigmented lesions were like melanoacanthoma, nevus and melanoma were considered under differential diagnosis made. Blood investigations and radiographic features did not reveal any significant findings. Incisional biopsy of the lesion was performed and sent for histopathological examination.

Haematoxylin and eosin-stained sections showed invasion of the connective tissue stroma by sheets and islands of pleomorphic epithelioid, spindle cell atypical melanocytes containing brownish to black pigment in the cytoplasm.(Fig.2) Further this was confirmed immunohistochemically by using HMB-45 (Fig 3) ; MelaninA, (Fig. 4), S-100 (Fig, 5 ), PCK, Ki67 (Fig 6) and LCA (Fig. 7) which showed strong positivity of the tumour cells. Immunohistochemical report shows Melanin-A(+), HMB45(+), S-100(+) ,PCK(-), LCA(-), Ki67(+). Thus, the lesion was diagnosed as melanoma.

A complete blood cell count, biochemical analysis, and urine analysis were insignificant and within normal limits. CECT revealed a well-defined in-homogenously enhancing hypodense nodule in the right maxillary region with no bony erosion.

Based on the clinical examination, radiologic and histopathological features a final diagnosis of malignant melanoma was arrived. Medical information was provided to the patient and his family regarding the diagnosis, staging, therapeutic options and prognosis. With diagnosis of primary malignant melanoma of gingiva, surgical intervention was performed with the gingival lesion along the right maxillary alveolar process including teeth was resected. Reconstruction of the defect was done with buccal fat pad flap. The postoperative recovery was found to be uneventful. Histopathology confirmed malignant melanoma with $2 \mathrm{~mm}$ tumor thickness with. The patient was under follow up with no recurrence.

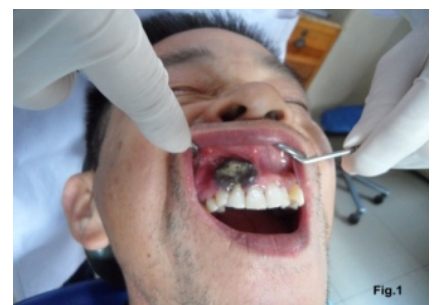

Figure 1: Intraoral photograp showing blackish brown lesion in relation to labial gingiv

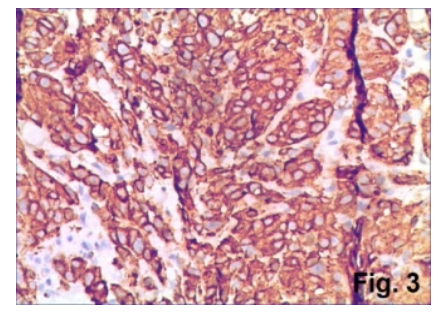

Figure 3: Immunohistochemical staining with HMB-45 antibody that stains the cytoplasm of the epithelioid cells

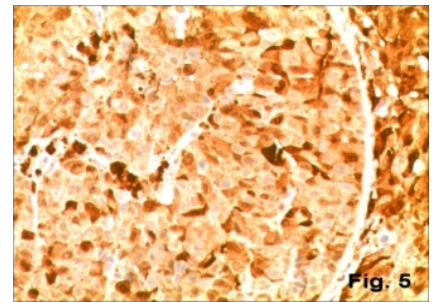

Figure 5: Immunohistochemical staining with S-100

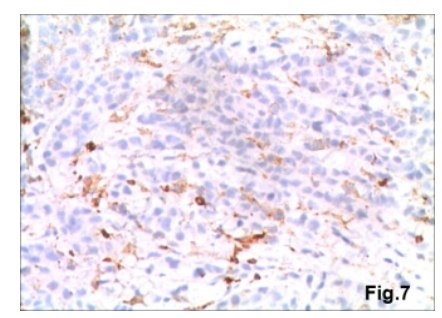

Figure 7: Immunohistochemica staining with LCA

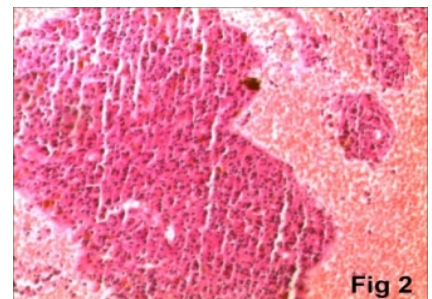

Figure 2: The hematoxylin and eosinstained section shows melanoma with invasive patter showing large cells with pleomorphic vesicular nucleus and brown pigment $(\times 40)$

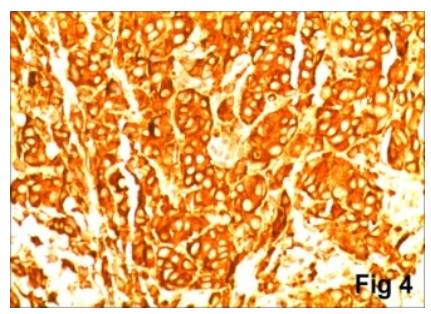

Figure 4: Immunohistochemical staining with Melan-A antibody showing cytoplasmic staining

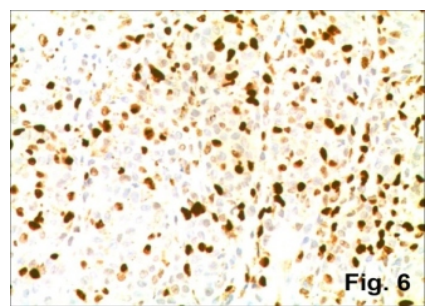

Figure 6: Immunohisto chemical staining with $\mathrm{Ki67}$ 


\section{DISCUSSION}

Oral malignant melanoma is an extremely rare neoplasm of melanocytes which was reported by Weber in $1856 .^{4}$ It is biologically an aggressive neoplasm with a poorer prognosis than its cutaneous counterpart. The etiology of malignant melanoma remains elusive. The risk factors for the development of melanoma include UV radiation, skin and hair color, numerous freckles, tendency to burn and tan poorly, PUVA therapy, tanning salons, presence of nevi (numerous, large, atypical), xeroderma pigmentosum, immuno supression, denture irritation, exposure to tobacco, chemicals, petroleum and printing products. Primary oral melanomas originated either from a nevus or pre-existing pigmented lesion currently most thought to arise de novo. In our case, patient was not exposed to above factors the possible etiology may be de novo. The genetic factors include mutations of the gene CDKN2A (Cyclin-Dependent Kinase Inhibitor 2A), encoding the tumor suppressor protein p16 located on chromosome 9p21 which confers susceptibility to familial malignant melanoma. Other genes include RB1, CDK4gene on chromosome 12q15, RB1 and PTEN/MMAC1.

Melanomas occur in all ages between 7 to 90 years and are most prevalent in the 5 th decade. Males are more commonly affected than females. The most common sites in the head and neck region include conjunctiva, sinonasal cavity, oral cavity, pharynx, larynx and upper oesophagus (in decreasing order). In the oral cavity $80 \%$ cases involves the gingiva and palate. Occurrence in the mandible, buccal mucosa, lips tongue and floor of mouth is not uncommon. ${ }^{7}$ In our case also, the male patient presented lesion in the $5^{\text {th }}$ decade of life, which were in the anterior maxillary gingiva. The findings were very similar to the published article in the different journals. That supports the diagnosis as malignant melanoma.

The differential diagnosis of OMM include of oral melanotic macule, melanoplakia, pituitary-based Cushings syndrome, post-inflammatory pigmentation, melanoacanthoma, amalgam tattoo, Addisons disease, Peutz-Jeghers syndrome and melanocytic nevi. ${ }^{8}$ But our patient did not have any other signs or symptoms suggesting any of these lesions.

Melanoma shows wide spectrum of histo-pathological features which are confused with mesenchyme, epithelial and neural tumors, S-100 and HMB-45 are more frequently expressed than Melan-A and these markers are helpful to confirm the diagnosis. ${ }^{9}$ Our case showed positive for HMB45, Melanin-A,S-100,Ki67.

The Clarks grading system assessing the depth of invasion and Breslow measuring the thickness of tumour from the surface of the epidermis to the greatest depth of the tumour have no validation as prognostic factors in OMM due to rarity of the lesion and absence of a true dermis in the oral cavity. However, a simple TNM staging with prognostic value have three stages.

It has always been suggested that incision into malignant neoplasm during incisional biopsy could result in accidental dissemination of malignant cells within adjacent tissues or blood or lymphatic stream with subsequent risk of local recurrence or regional or distant metastasis. Rampen et al. and Austin et al. did find a somewhat reduced survival rate in patients with melanoma who had incisional biopsies but against the studies done by Lederman and Sober where they found no correlation in patient's prognosis with incisional and excisional biopsies. Recurrences may occur even after 1015 years after primary therapy. Distant metastasis to the lungs, brain, liver, and bones are frequently observed.

It is well known that early diagnosis and treatment of melanoma can reduce mortality. If diagnosed early when the malignant cells are limited to the epithelium or invasion is minimal. Melanoma is either $100 \%$ curable by excision (for in situ lesion) or is associated with a 5-year survival rate of $95 \%$ (for lesions $<1 \mathrm{~mm}$ in thickness and without ulceration). In contrast, the 5-year survival rate for cutaneous melanomas $>4$ $\mathrm{mm}$ thickness with ulceration is only $45 \%{ }^{12}$

Poor prognosis of melanoma may be due to early invasion of deeper structures due to proximity of bone and muscles increasing likelihood of metastasis. Rich vascular supply of oral cavity further aids in dissemination of melanoma. ${ }^{13} \mathrm{~A}$ variety of serological markers like serum lactate dehydrogenase (LDH), melanoma-inhibiting activity (MIA), S100B and vascular endothelial growth factor (VEGF) are available for melanoma. Elevated levels of LDH and MIA are associated with more advance stages and poorer prognosis. These markers are useful in monitoring the patient's clinical course of the disease and response to therapy.

The treatment options for mucosal melanomas of oral cavity include surgery, radiation and adjuvant chemotherapy and/or immunotherapy. Surgery is the mainstay of treatment, but it may be challenging, depending on the anatomic location within the oral cavity and extent of the tumor. Optional treatment of clinically negative neck nodes with neck dissection or radiotherapy was recommended because of the high risk of subclinical disease. Although melanoma is classically not radiosensitive, some authors have described improved survival and local control with postoperative radiotherapy. ${ }^{14}$ Despite the improvement of surgical techniques and the introduction of new chemotherapeutic agents, prognosis of this malignancy remains poor so close patient monitoring is imperative to check for recurrence.. In our case, early diagnosis helped to perform early surgery with no recurrence was found eight month postoperative.

\section{CONCUSION}

The purpose of this manuscript is to emphasize on early diagnosis and to maintain high index of suspicion for those pigmented lesions occurring in the high risk sites such as palate and maxillary gingiva. 


\section{REFERENCES}

1. Ullah H, Vahiker S, Singh M, Baig M. Primary malignant mucosal melanoma of the oral cavity: A case report. Egypt J Ear Nose Throat Allied Sci. 2010;11:4850.

2. Dimitrakopoulos I, Lazaridis N, Skordalaki A. Primary malignant melanoma of the oral cavity. Report of an unusual case. Aust Dent J. 1998;43:37981. [PubMed]

Http://dx.doi.org/10.1111/j.1834-7819.1998.tb00194.x

Http://dx.doi.org/10.1111/j.1834-7819.1998.tb00191.x PMid:9973703

3. Tanaka N, Mimura M, Ichinose S, Odajima T. Malignant melanoma in the oral region: Ultrastructural and immunohistochemical studies. Med Electron Microsc. 2001;34:198205. [PubMed]

http://dx.doi.org/10.1007/s007950100016 PMid:11793197

4. Vikey AK, Vikey D. Primary malignant melanoma, of head and neck: A comprehensive review of literature. Oral Oncol. 2012;48:399403. [PubMed]

Http://dx.doi.org/10.1016/j.oraloncology.2011.12.014

PMid:22265336

5. Gondivkar SM, Indurkar A, Degwekar S, Bhowate R. Primary oral malignant melanoma: A case report and review of the literature. Quintessence Int. 2009;40:416. [PubMed] PMid:19159022

6. Rajendran R, Sivapathasundharam B. Shafer, Hine, Levy's Shafer's Text Book of Oral Pathology. 6th ed. Vol. 80. India: Elsevier; 2009. Benign and malignant tumors of the oral cavity; p. 218

7. Manganaro A, Hammond H, Dalton M, Williams TP. Oral melanoma: Case report and review of literature. Oral Surg Oral Med Oral Pathol Oral Radiol Endod. 1995;85:6706. [PubMed] http://dx.doi.org/10.1016/S1079-2104(05)80250-1
8. Femiano F, Lanza A, Buonaiuto C, Gombos F, Di Spirito F, Cirillo N. Oral malignant melanoma: A review of the literature. $J$ Oral Pathol Med. 2008;37:3838. [PubMed] Http://dx.doi.org/10.1111/j.1600-0714.2008.00660.x PMid:18284541

9. Devi P, Bhovi T, Jayaram RR, Walia C, Singh S. Malignant melanoma of the oral cavity showing satellitism. J Oral Sci. 2011;53:23944. [PubMed]

http://dx.doi.org/10.2334/josnusd.53.239 PMid:21712630

10. Peckitt NS, Wood GA. Malignant melanoma of oral cavity. Oral Surg Oral Med Oral Pathol. 1990; 70:1614. [PubMed] http://dx.doi.org/10.1016/0030-4220(90)90111-5

11. Meleti M, Leemans CR, Mooi WJ, Vescovi P, van der Waal I. Oral malignant melanoma: A review of the literature. Oral Oncol. 2007;43:11621. [PubMed]

Http://dx.doi.org/10.1016/j.oraloncology.2006.04.001 PMid:16931116

12. Auluck A, Zhang L, Desai R, Rosin MP. Primary malignant melanoma of maxillary gingiva A case report and review of the literature. J Can Dent Assoc. 2008;74:36771. [PubMed] PMid:18538076

13. Wanjari PV, Warhekar AM, Wanjari SP, Reddy V, Tekade S, Shrivastava P. Primary Oral Malignant Melanoma. J Indian Acad Oral Med Radiol. 2011;23:769.

http://dx.doi.org/10.5005/jp-journals-10011-1098

14. Kingdom TT, Kaplan MJ. Mucosal melanoma of the nasal cavity and paranasal sinuses. Head Neck. 1995;17:184189. [PubMed] Http://dx.doi.org/10.1002/hed.2880170303 PMid:7782202 